\title{
Masked orbital abscess in Wegener's granulomatosis
}

\begin{abstract}
Two patients with chronic Wegener's granulomatosis presented with worsening proptosis and visual acuity. Both patients had been maintained on long-term corticosteroids, which led to masking of the signs of orbital sepsis with potentially life-threatening implications.

Eye (2007) 21, 246-248. doi:10.1038/sj.eye.6702211; published online 13 January 2006
\end{abstract}

Keywords: Wegener's granulomatosis; orbit; abscess; immunosuppression

Wegener's granulomatosis is a rare autoimmune vasculitis characterized by a generalized disease, associated with a necrotizing glomerulonephritis and high mortality, and by a more limited form, associated with respiratory tract, nasal, oral, or orbital inflammation. ${ }^{1-3}$ We describe two patients with Wegener's granulomatosis on long-term immunosuppressants, who presented with worsening proptosis, in whom the underlying chronic orbital abscess was not immediately evident. ${ }^{4,5}$

Service, Western Eye Hospital, London, UK

${ }^{2}$ Institute of Ophthalmology, London, UK

Correspondence: JM Olver, Consultant

Ophthalmologist,

Oculoplastic and Orbital Surgeon, Western Eye Hospital, Marylebone Road, London NW1 5YE, England, UK.

Tel: +44 207886 3258;

Fax: + 442078863259 .

E-mail: janeolver@

aol.com

Received: 6 August 2005 Accepted in revised form: 8 October 2005

Published online:

13 January 2006

\section{Case Series}

\section{Case 1}

A 72-year-old Caucasian female presented with left orbital pain and intermittent diplopia. She had been treated for Wegener's granulomatosis for 7 years, and maintained on oral prednisolone $10 \mathrm{mg}$ daily. Her right corrected visual acuity was $6 / 9$, and left $6 / 18$. She developed left nonaxial proptosis of $2 \mathrm{~mm}$ and grossly reduced upgaze. Intraocular pressures were 25 for the right eye and 37 for the left eye. She was systemically well. Apyrexial, antineutrophil cytoplasmic antibody (ANCA) testing was negative and erythrocyte sedimentation rate (ESR) was 13 . CT revealed a left orbit soft tissue lesion extending from the posterior surface of the globe to the optic foramen (Figure 1). A provisional diagnosis of an exacerbation of Wegener's granulomatous was made by the physicians and treatment commenced with high dose corticosteroids (60 mg prednisolone/once day). Proptosis, ocular pain, and systemic health deteriorated over a 2-week period and she was referred to the Oculoplastic and Orbital Service. Trans-conjunctival orbital exploration identified and drained an extensive loculated orbital abscess with rapid reversal of clinical symptoms. Histopathological analysis of the orbital biopsy showed chronic inflammation with a granulamatous component. There was necrosis and fibrosis, with recanalised vessels (Figure 2); however, no active vasculitis was present. At 12 months postoperation, the patient remained well with left visual acuity of $6 / 6$ and normal intraocular pressures, on a low dose of corticosteroids.

\section{Case 2}

A 62-year-old Caucasian female presented with a 2-week history of increasing right neck swelling and proptosis. She had been treated for Wegener's granulomatosis for 14 years and maintained on $10 \mathrm{mg}$ prednisolone once daily. Visual acuity was 6/24 in the right eye and 6/12 in the left eye. Examination showed $5 \mathrm{~mm}$ of right nonaxial proptosis with gross restriction of right ocular movements and a 35-dioptre exotropia. Colour vision, intraocular pressures, and fundoscopy were normal. CT orbital imaging showed a low attenuation mass in the right medial, supero-medial intra- and extraconal orbit with no evidence of sinusitis. Investigations revealed a mildly elevated CRP (36), normal white cell count, and negative c-ANCA.

A provisional diagnosis of the exacerbation of Wegener's granulomatous was made and her physicians commenced treatment with systemic corticosteroids (prednisolone $50 \mathrm{mg}$ /once day) and cytotoxics (azothiaprine $50 \mathrm{mg}$ /once day 


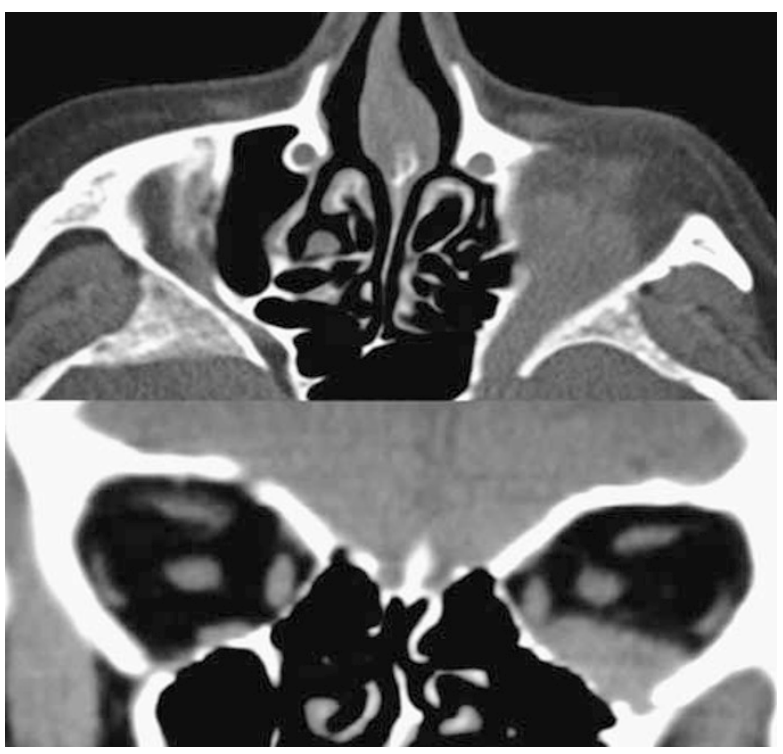

Figure 1 Axial and coronal orbital CT of patient 1. Left low attenuation lesion extending from the inferior mid-orbit to the orbital apex, with involvement of inferior and medial recti.

and cyclophosphamide $100 \mathrm{mg}$ /once day). However, over a 2-week, period the patient's health rapidly deteriorated with sepsis and she suffered three cardiac arrests. Blood cultures grew Streptococcus milleri and the patient was started on a broad spectrum of antibiotics (Cefradine1 g 6 h, Teicoplanin $400 \mathrm{mg} 12 \mathrm{~h}$,

Benzylpenicillin $1.2 \mathrm{~g} 6 \mathrm{~h}$, and Metronidazole $500 \mathrm{mg} 8 \mathrm{~h}$ ).

Right proptosis and chemosis progressed over a 2-week period. She was referred to the Oculoplastic and Orbital Service. Upon trans-conjunctival orbital exploration, a medial loculated orbital abscess was drained and histopathological analysis of orbital biopsy showed nonspecific orbital fibrosis with chronic inflammation consistent with a multiloculated abscess. Postoperatively, the right visual acuity was $6 / 9$ and 3 years on the patient remains well with no orbital problem and remains on $15 \mathrm{mg}$ prednisolone/daily.

\section{Comment}

Orbital and adnexal Wegener's granulomatosis is rare and has a variable clinical presentation and may have an indolent course. A retrospective survey of Australian Oculoplastic surgeons reviewed the findings of 29 cases. Proptosis occurred in almost 70\%, nasolacrimal duct obstruction and epiphora in 52\%, limited eye movements in $52 \%$, lid erythema and oedema in $31 \%$, but none had chronic orbital abscess. ${ }^{2}$

Our two cases emphasise that Wegener's patients maintained on long-term immunosuppressants may pose a management dilemma as proptosis and orbital signs can result from exacerbation of the primary
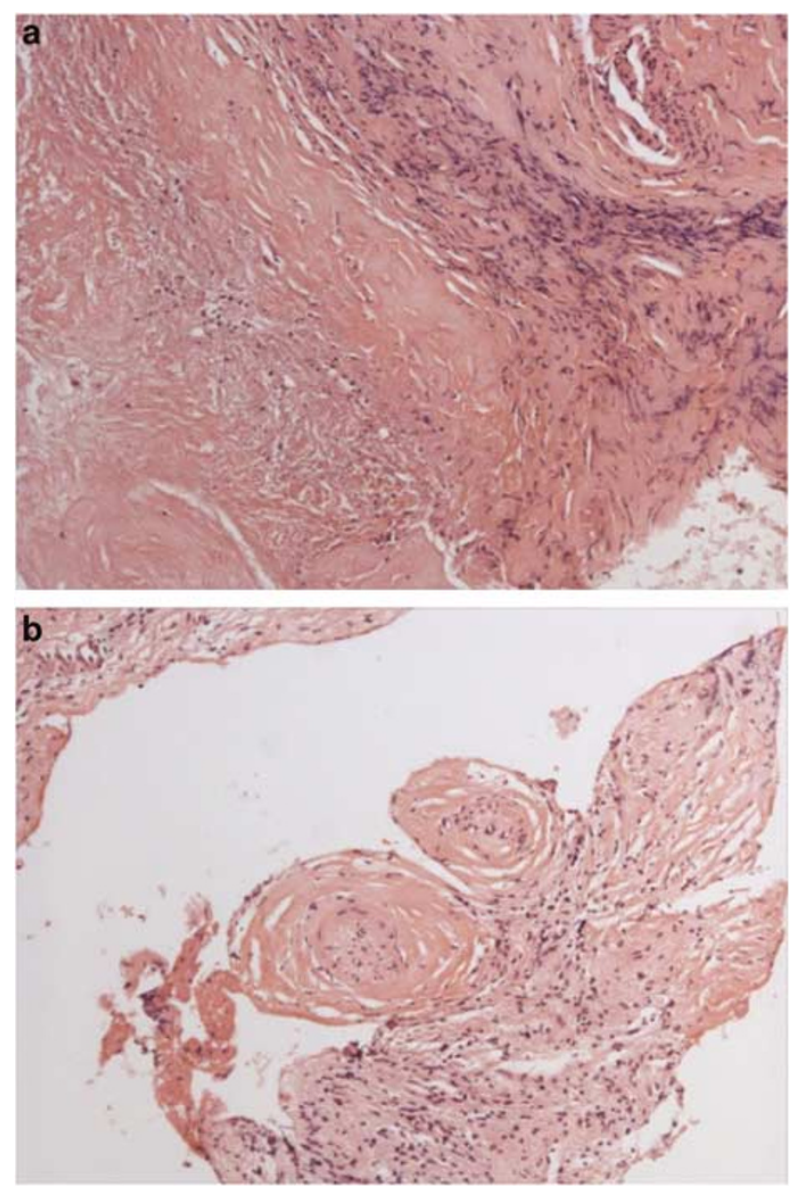

Figure 2 Histopathology from the orbital biopsy of patient 1 . Medium power photomicrographs showing chronic inflammatory cell infiltrates with areas of necrosis (a) and fibrotic vessels (b) (Haematoxylin and Eosin $\times 100$ ).

inflammatory disorder or secondary infection with chronic orbital abscess secondary to immunosuppression. Treatment with further systemic immunosuppression may lead to a masking of the signs of sepsis and even result in a fulminating life threatening sepsis, as in our second case. With a loculated or localised infection, inflammatory markers (ESR, CRP) may be within normal limits, and the systemic features of infection may be masked. Blood cultures were not taken in patient 1 (apyrexial), and were taken in patient 2 as the patient's health deteriorated, which led to the diagnosis. Orbital sepsis is an uncommon condition characterized by infection of the soft tissues of the orbit. In the absence of pyrexia and elevated inflammatory markers, positive signs include orbital proptosis and visual impairment, lid oedema secondary to reduced venous outflow and cranial nerve palsies. Without treatment, orbital abscess formation or cavernous sinus thrombosis may occur.

These cases suggest that clinicians should have a high suspicion of chronic orbital abscess in Wegener's patients 
with worsening proptosis. Radiological investigation with CT scanning is essential, and we propose a low threshold for orbital exploration and biopsy.

\section{References}

1 Harman LE, Margo CE. Wegener's granulomatosis. Surv Ophthalmol 1998; 42(5): 458-480.

2 Woo TL, Francis IC, Wilcsek GA, Coroneo MT,

McNab AA, Sullivan TJ. Australasian orbital and adnexal
Wegener's granulomatosis. Ophthalmology 2001; 108(9): 1535-1543.

3 Haynes BF, Fishman ML, Fauci AS, Wolff SM. The ocular manifestations of Wegener's granulomatosis. Fifteen years experience and review of the literature. Am J Med 1977; 63(1): 131-141.

4 Kalina PH, Lie JT, Campbell RJ, Garrity JA. Diagnostic value and limitations of orbital biopsy in Wegener's granulomatosis. Ophthalmology 1992; 99(1): 120-124.

5 Bullen CL, Liesegang TJ, MacDonald TJ, DeRemee RA. Ocular complications of Wegener's granulomatosis. Ophthalmology 1983; 90: 279-290. 